\title{
Efecto Tóxico del Extracto Acuoso de Ruta graveolens del Norte de México sobre el Hígado de Rata Wistar
}

\author{
Toxic Effect of Aqueous Extract of Ruta graveolens from \\ the North of Mexico on the Liver of Wistar Rat
}

\author{
Luis B. Serrano-Gallardo*; Adolfo Soto-Domínguez**; Pablo Ruiz-Flores*; Martha P. Nava-Hernández*; \\ Javier Morán-Martínez***; Rubén García-Garza** \& Edith F. Martínez-Pérez*
}

\begin{abstract}
SERRANO-GAllardo, L. B.; SOTO-DOMÍNGUEZ, A.; RUIZ-FLORES, P.; NAVA-HERNÁNDEZ, M. P.; MORÁNMARTÍNEZ, J.; GARCÍA-GARZA, R. \& MARTÍNEZ-PÉREZ, E. F. Efecto tóxico del extracto acuoso de Ruta graveolens del Norte de México sobre el hígado de rata wistar. Int. J. Morphol., 31(3):1041-1048, 2013.
\end{abstract}

RESUMEN: A Ruta graveolens se le atribuyen propiedades benéficas en medicina tradicional, sin embargo se ha demostrado que también puede producir efectos adversos. A la fecha no existe evidencia que ratifique que $R$. graveolens es totalmente inofensiva, por este motivo el objetivo de este estudio fue determinar el efecto tóxico del extracto acuoso de las hojas de esta especie vegetal en hígado de rata. Se utilizaron 25 ratas Wistar y se dividieron en 5 grupos $(\mathrm{n}=5)$. El grupo 1 correspondió al grupo control negativo, el grupo 2 o control positivo se trató con $100 \mathrm{mg}$ de dexametasona/kg/día. Los grupos 3 y 4 se trataron con 30 y 100 mg de extracto de $R$. graveolens respectivamente. Al grupo 5 se le administraron $100 \mathrm{mg}$ de dexametasona/kg/día combinado con $100 \mathrm{mg}$ de extracto de $R$. graveolens/ $\mathrm{kg}$ /día. Las administraciones se realizaron vía intraperitoneal por tres días. Los animales se sacrificaron por dislocación cervical. Las muestras de hígado se fijaron en formalina y posteriormente se incluyeron en parafina. Se obtuvieron cortes histológicos de 5 micras de grosor que se tiñeron con Hematoxilina-Eosina para la evaluación histológica con microscopia de luz. Los resultados demuestran por primera vez que la exposición al extracto acuoso de $R$. graveolens induce alteraciones morfológicas en el hígado de ratas Wistar. Estas alteraciones se observaron de manera leve en el grupo 3 y se incrementaron en el grupo 4. Destacó que el mayor daño se observó en el grupo 5. Se concluye que el extracto acuoso de $R$. graveolens resultó tóxico, sin embargo es necesario realizar estudios adicionales con el fin de caracterizar otros efectos toxicológicos que soporten el riesgo del uso de $R$. graveolens en medicina tradicional a largo plazo.

PALABRAS CLAVE: Ruta graveolens; Extracto acuoso; Toxicidad hepática; Ratas Wistar.

\section{INTRODUCCIÓN}

El uso de la medicina herbolaria está creciendo de manera progresiva como alternativa a la práctica terapéutica actual (Agbonon et al., 2010). Cuando menos en alguna ocasión las plantas medicinales se han utilizado por el 70-80\% de la población a nivel mundial como aditivo alimenticio o para el tratamiento de diversas enfermedades (Libman et al., 2006; Shanmugan et al., 2012). Las plantas se han empleado para el tratamiento de las enfermedades infecciosas bacterianas, virales, parasitarias, fúngicas, inflamatorias, espasmos entre muchos otros padecimientos. Actualmente cerca del $25 \%$ de los fármacos prescritos por los médicos, tuvieron su origen en las plantas (Alonso-Castro et al., 2012).
Ruta graveolens es una planta nativa de Europa, pero actualmente se encuentra distribuida en todo el mundo (Raghav et al., 2006). Es un arbusto conocido popularmente como ruda, crece aproximadamente un metro de altura, posee un tallo muy ramificado y hojas verdes grisáceas (Harat et al., 2008). Se le reconocen propiedades farmacológicas como antinociceptivo, antiinflamatorio, fungicida, herbicida y citotóxico (Oliva et al., 2003; González-Trujano et al., 2006; Ratheesh et al., 2009; Varamini et al., 2009). Su efecto abortivo ha sido reportado en diferentes países como Brasil, la India y México (Rahim et al., 2010). La aplicación principal en infusión de té es el alivio de los cólicos menstruales por sus propiedades antiespasmódicos (Seak \& Lin, 2007).

\footnotetext{
* Departamento de Bioquímica y Farmacología. Centro de Investigación Biomédica, Universidad Autónoma de Coahuila, Torreón, Coahuila, México.

** Departamento de Histología, Facultad de Medicina, Universidad Autónoma de Nuevo León, Monterrey, Nuevo León, México.

**** Departamento de Biología Celular y Ultraestructura. Centro de Investigación Biomédica, Universidad Autónoma de Coahuila, Torreón, Coahuila, México.
} 
SERRANO-GALLARDO, L. B.; SOTO-DOMÍNGUEZ, A.; RUIZ-FLORES, P.; NAVA-HERNÁNDEZ, M. P.; MORÁN-MARTÍNEZ, J.; GARCÍA-GARZA, R. \& MARTÍNEZ-PÉREZ, E. F. Efecto tóxico del extracto acuoso de Ruta graveolens del Norte de México sobre el hígado de rata wistar. Int. J. Morphol., 31(3):1041-1048, 2013.

De $R$. graveolens se han aislado más de 120 compuestos entre los que se encuentran el aceite volátil 2undecanona como su mayor componente (50-90\%), 2hepatonol, 2-nonanona, limoneno, pineno, ácido anísico, fenol, guiacol y otros. Rutina y otros flavonoides como quercetina; cumarinas como bergapteno, dafnoretina, isoimperatorina, naftoherniarina, psoraleno, pangelina, rutamarina, rutarina, escopoletina y umbeliferona; alcaloides como arborinina, fagarina, graveolina, cocusaginina, rutacridina, 1-hidroxi-3-metoxi-N-metilacridona; lignanos en la raíz como savinina yhelioxantina (Ratheesh et al.).

Los compuestos derivados de las cumarinas se han utilizado en neurología para el tratamiento sintomático de enfermedades desmielinizantes como la esclerosis múltiple (Orlita et al., 2008). La rutina se vende en farmacias como constituyente medicinal contra la fragilidad capilar, vermífugo intestinal y como antídoto para las toxinas de serpiente y escorpión (Freire et al., 2010), además se ha utilizado para la psoriasis leucodérmica, linfomas cutáneos, artritis reumática y contra el cáncer (Diwan \& Malpathak, 2009). Las furanocumarinas se han empleado para tratar la leucoderma, vitíligo, linfomas cutáneos y como agente anti-HIV (Diwan \& Malpathak, 2010).

Existe la creencia de que las plantas medicinales son innocuas, pero estudios previos han demostrado los efectos tóxicos que muestran algunas especies utilizadas en medicina tradicional (Inamul, 2004). Estudios farmacológicos han confirmado los efectos adversos de la $R$. graveolens, como por ejemplo: fototoxicidad, genotoxicidad y mutagenicidad (Freire et al.). El objetivo del presente trabajo fue determinar el efecto tóxico del extracto acuoso de $R$. graveolens del norte de México sobre el hígado de ratas Wistar.

\section{MATERIAL Y MÉTODO}

Preparación del extracto acuoso de $\boldsymbol{R}$. graveolens. Se colectaron aproximadamente $5 \mathrm{~kg}$ de planta fresca de $R$. graveolens en el Norte de México en las coordenadas 25.53607 N y 103.52117 O. El material vegetal se secó durante 10 días a temperatura ambiente, posteriormente las partes aéreas se molieron hasta obtener un polvo fino. De este polvo se pesaron $100 \mathrm{~g}$ y se le agregaron 1000 $\mathrm{mL}$ de agua destilada, la mezcla se calentó a $100^{\circ} \mathrm{C}$ y se agitó por una hora. Luego se filtró con papel Whatman $\mathrm{N}^{\mathrm{o}} 1$ con bomba al vacío. Finalmente el filtrado se llevó a sequedad en estufa a $40^{\circ} \mathrm{C}$ para obtener el extracto seco que se almacenó a $5^{\circ} \mathrm{C}$ hasta su utilización.

Animales de experimentación y grupos del estudio in vivo. En este estudio se emplearon 25 ratas Wistar machos de 11 semanas de edad y peso de 250-300 g. Siete días previos al tratamiento se mantuvieron bajo condiciones estándar de laboratorio, temperatura $24^{\circ} \mathrm{C}$, ciclos luz/oscuridad de 12 horas, humedad relativa de $45 \%$ con libre acceso al pellet Teklad® (18\% proteína) y agua $\mathrm{ad}$ libitum. Ningún animal fue reemplazado durante el estudio. El protocolo experimental fue aprobado por el Comité de Bioética de la Facultad de Medicina de la Universidad Autónoma de Coahuila.

Las ratas se dividieron en cinco grupos $(n=5)$. El grupo 1 correspondió al control negativo, al grupo 2 se le administraron $100 \mathrm{mg}$ de dexametasona disuelta en aceite de maíz/kg/día vía intraperitoneal (i.p.), este grupo se consideró como control positivo. Los grupos 3 y 4 se trataron con 30 y $100 \mathrm{mg}$ de extracto de $R$. graveolens disuelto en solución fisiológica $0.9 \% / \mathrm{kg} /$ día vía i.p. respectivamente. Finalmente al grupo 5 se le administraron $100 \mathrm{mg}$ de dexametasona $/ \mathrm{kg} /$ día combinado con $100 \mathrm{mg}$ de extracto de $R$. graveolens $/ \mathrm{kg} /$ día.

Las administraciones de dexametasona o del extracto de $R$. graveolens se realizaron por tres días consecutivos. Después de la última administración los animales se mantuvieron en ayuno durante 24 horas, y se sacrificaron por dislocación cervical. De cada animal se disecó el hígado, y se tomaron muestras representativas que se fijaron en formalina al $10 \%$.

Análisis Histológico. Las muestras de hígado colectadas se procesaron por la técnica histológica convencional hasta su inclusión en bloques de parafina. En el micrótomo se obtuvieron cortes histológicos de 5 micras de grosor que se tiñeron con Hematoxilina-Eosina para la evaluación histológica con microscopio de luz.

Análisis morfométrico. En este estudio también se realizó un análisis cuantitativo dividiendo el parénquima del hígado en acinos hepáticos o de Rappaport. Se analizaron 8 campos consecutivos con el objetivo de inmersión en aceite $(100 \mathrm{X})$, posteriormente se cuantificaron los hepatocitos normales diferenciándolos de los que mostraron alteraciones en las 3 zonas descritas para el acino hepático, esto se realizó por triplicado. De los valores obtenidos se obtuvieron valor promedio y desviación estándar (DE) y se analizaron estadísticamente con la prueba de $\mathrm{t}$ de student para un valor de significancia $\mathrm{p} \leq 0,05$. 
SERRANO-GALLARDO, L. B.; SOTO-DOMÍNGUEZ, A.; RUIZ-FLORES, P.; NAVA-HERNÁNDEZ, M. P.; MORÁN-MARTÍNEZ, J.; GARCÍA-GARZA, R. \& MARTÍNEZ-PÉREZ, E. F. Efecto tóxico del extracto acuoso de Ruta graveolens del Norte de México sobre el hígado de rata wistar. Int. J. Morphol., 31(3): 1041-1048, 2013.

\section{RESULTADOS}

Cambio del peso corporal durante el tratamiento. Durante el tratamiento se evaluó el cambio del peso de los animales para relacionarlo con el peso del hígado al final del experimento. No hubo cambio del peso corporal entre los grupos. En la Tabla I se muestran los resultados obtenidos con la prueba de ANOVA para peso corporal y del hígado, encontrando diferencia significativa.

Tabla I. Resultados obtenidos de la prueba de ANOVA para peso corporal e hígado $(\mathrm{n}=5)$.

\begin{tabular}{lc}
\hline & Valor de $\mathrm{p}$ \\
\hline Peso del Hígado & $0,003^{*}$ \\
Peso Corporal & $0,714 \&$ \\
\hline * Diferencia significativa respecto al control $(\mathrm{p}<$ \\
$0,05)$. \& Análisis mediante Kruskal Wallis.
\end{tabular}

En la Tabla II se muestran los resultados obtenidos mediante la prueba post hoc de Tukey determinando que existe diferencia significativa del grupo control positivo con dexametasona ( $\mathrm{p}=0,014$; IC 95\%: $-5,45,-0,51)$ y este último con el grupo tratado en dosis de $30 \mathrm{mg} / \mathrm{Kg} /$ día de extracto acuoso de $R$. graveolens ( $\mathrm{p}=0,02 ; \mathrm{IC} 95 \%$ : 1,25 - 6,19).

Análisis histológico. En todos los grupos de estudio, macroscópicamente el hígado no mostró presencia de hemorragia o cambio en la coloración. En el análisis histológico de las muestras del grupo 1 (control negativo) se observó una histología normal para este órgano: láminas de hepatocitos mononucleados o binucleados, con núcleos eucromáticos, citoplasma homogéneo y límites celulares bien definidos. Las láminas se observaron dispuestas de manera radial con respecto a la vena central, separadas por capilares sinusoidales con eritrocitos en su interior de características normales. Los espacios porta no mostraron alteraciones (Fig. 1A).

El grupo 2, mostró daño generalizado, caracterizado por pérdida de la arquitectura normal de los lobulillos hepáticos con desorganización de las láminas de hepatocitos. En los hepatocitos se observó aumento del tamaño celular, abundantes vacuolas en el citoplasma por la aparente extracción de lípidos o esteatosis microvesicular, y signos francos de necrosis, también se observó dilatación de los capilares sinusoidales (Fig. 1B).

En el grupo 3 se observó ligera congestión vascular y hepatocitos con aumento de tamaño. Destaca que en este grupo algunos hepatocitos mostraron signos de necrosis: extracción total o parcial del citoplasma y núcleos picnóticos, así como sugestivos de apoptosis como condensación de la cromatina alrededor de la envoltura nuclear, y condensación del citoplasma (Fig. 1C).

En el grupo 4, se observó aumento del daño observado en el grupo 3, con un mayor número de hepatocitos con signos de necrosis y apoptosis. En este grupo también se observaron focos de hepatocitos con abundantes vacuolas en su citoplasma por la extracción de lípidos, focos de hemorragia y congestión sinusoidal, así como zonas con infiltrado de células mononucleares (Fig. 1D).

En el grupo 5 se observó acentuación de las alteraciones descritas en los grupos 2, 3 y 4 (Fig. 1E).

Análisis morfométrico. En el análisis morfométrico de los acinos hepáticos en el grupo 1 se observaron escasos hepatocitos dañados por campo (zona 1: 0,333 \pm 0,577, zona 2: $0,166 \pm 0,288$ y zona 3: $0,1 \pm 0,173)$. En el grupo 2 se observó una mayor cantidad de hepatocitos alterados por campo en las 3 zonas de estudio (zona 1: 21,437 $\pm 2,032$, zona 2: 15,562 $\pm 0,795$ y zona $3: 15,875 \pm 1,237)$.

En el grupo 3, solo se observó un número pequeño de hepatocitos dañados (zona 1: 1,333 $\pm 1,092$, zona 2: 1,583 \pm $1,134$ y zona $3: 0,708 \pm 0,144)$.

Sin embargo, en el grupo 4 se observó un daño acentuado en los hepatocitos (zona 1: 15,291 $\pm 2,943$, zona 2: 14,333 $\pm 4,889$ y zona $3: 14,583 \pm 5,789)$, similar al observado en el grupo 2. Destaca que en el grupo 5, el daño es aún mayor (zona 1: $27,666 \pm 1,404$, zona $2: 24,375 \pm 0,544$ y zona $3: 21,5 \pm$ $3,727)$. Tanto en los grupos $2,3,4$ y 5 se observó diferencia estadística significativa comparados con el grupo 1 (Fig. 2).

Tabla II. Efecto producido en el peso del hígado mediante la administración i.p.de extracto acuoso de $R$. graveolens $(\mathrm{n}=5)$ a.

\begin{tabular}{lcc}
\hline Tratamiento & Peso corporal $(\mathrm{g})$ & Peso del hígado $(\mathrm{g})$ \\
\hline Grupo 1 (control negativo) & $271,6 \pm 30,66$ & $8,30 \pm 0,71^{*}$ \\
Grupo 2 (100 mg de Dex./kg/día) & $277,2 \pm 36,72$ & $11,28 \pm 2,27^{*+}$ \\
Grupo 3 (30 mg de R.graveolens/Kg/día) & $299,6 \pm 10,69$ & $7,56 \pm 0,86+$ \\
Grupo 4 (100 mg de R.graveolens./Kg/día) & $280,8 \pm 22,99$ & $8,92 \pm 0,72$ \\
Grupo 5 (100 mg de R.graveolens $100 \mathrm{mg}$ de Dex./Kg/día) & $274,6 \pm 46,49$ & $9,60 \pm 1,26$ \\
\hline
\end{tabular}

a Los resultados son expresados como la media \pm DE

*Diferencia significativa respecto al grupo A con la prueba de Tukey Post Hoc $(\mathrm{p}<0,05)$.

+ Diferencia significativa respecto al grupo B con la prueba de Tukey Post Hoc $(\mathrm{p}<0,05)$. 

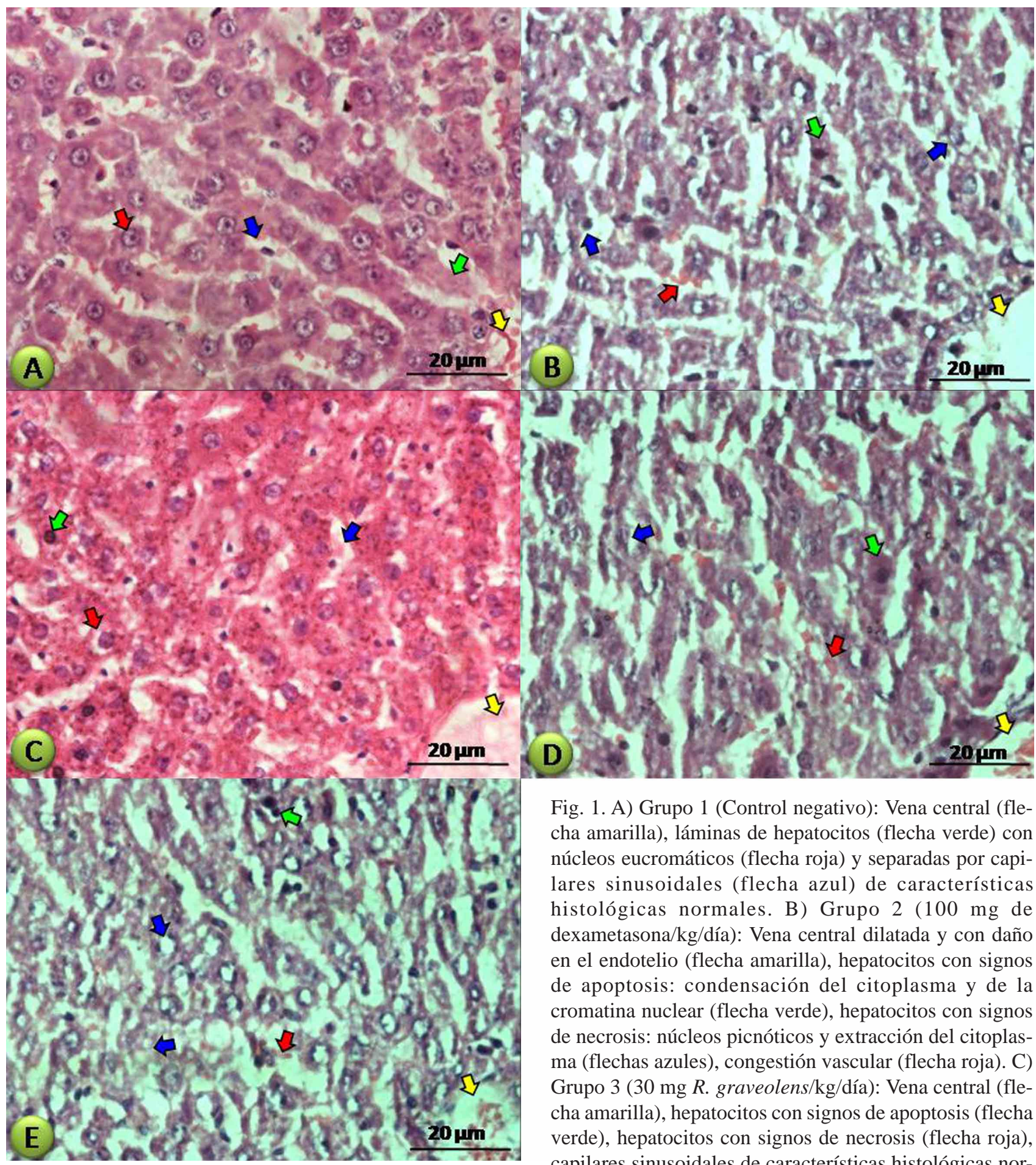

Fig. 1. A) Grupo 1 (Control negativo): Vena central (flecha amarilla), láminas de hepatocitos (flecha verde) con núcleos eucromáticos (flecha roja) y separadas por capilares sinusoidales (flecha azul) de características histológicas normales. B) Grupo $2(100 \mathrm{mg}$ de dexametasona $/ \mathrm{kg} /$ día): Vena central dilatada y con daño en el endotelio (flecha amarilla), hepatocitos con signos de apoptosis: condensación del citoplasma y de la cromatina nuclear (flecha verde), hepatocitos con signos de necrosis: núcleos picnóticos y extracción del citoplasma (flechas azules), congestión vascular (flecha roja). C) Grupo 3 (30 mg $R$. graveolens $/ \mathrm{kg} / \mathrm{día}$ ): Vena central (flecha amarilla), hepatocitos con signos de apoptosis (flecha verde), hepatocitos con signos de necrosis (flecha roja), capilares sinusoidales de características histológicas normales (flecha azul). D) Grupo 4 (100 mg $R$. graveolens $/ \mathrm{kg} /$ día): Congestión vascular en vena central (flecha amarilla), y sinusoides (flecha roja), hepatocitos con signos de apoptosis (flecha verde) y necrosis (flecha azul). Destaca la pérdida de la arquitectura normal y la presencia de daño generalizado. E) Grupo $5(100 \mathrm{mg} R$. graveolens $/ \mathrm{kg} / \mathrm{día}+100 \mathrm{mg}$ dexametasona/ $\mathrm{kg} /$ día): Acentuación de las alteraciones descritas en los grupos 2, 3 y 4: congestión vascular en vena central y el daño en las células endoteliales (flecha amarilla), dilatación de los sinusoides con daño en el endotelio (flecha roja), necrosis generalizada en hepatocitos (flechas azules), hepatocitos con signos de apoptosis (flecha verde). Hígado. Embebido en parafina, HE. Micrografía de luz. Barra $20 \mu \mathrm{m}$. 


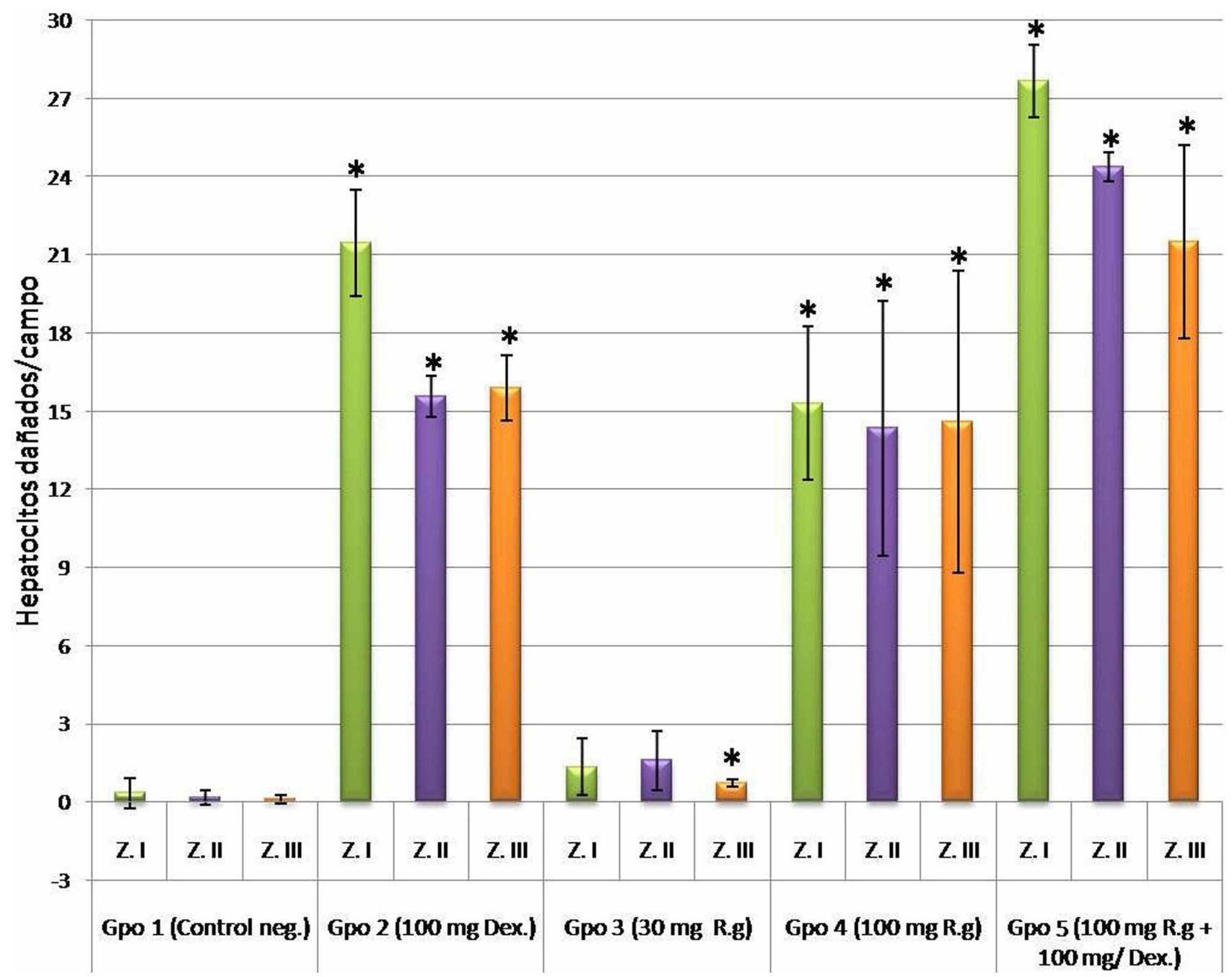

Fig. 2. Gráfica que muestra los resultados del análisis morfométrico en cada uno de los grupos de estudio. El análisis estadístico muestra que hay diferencia significativa entre $\operatorname{los}$ grupos $2,3,4$ y 5 vs. el grupo $1 .^{*} \mathrm{p} \leq 0,05$.

\section{DISCUSIÓN}

Los resultados del presente estudio demuestran por primera vez que la exposición aguda al extracto acuoso de $R$. graveolens del norte de México induce alteraciones morfológicas en el hígado de ratas Wistar. Estas alteraciones se observaron de manera leve al administrar una concentración de $30 \mathrm{mg}$ del extracto de $R$. graveolens $/ \mathrm{kg} / \mathrm{día}$ en tres días consecutivos. Las alteraciones se incrementaron con la administración de $100 \mathrm{mg}$ del extracto de $R$. graveolens $/ \mathrm{kg} / \mathrm{día}$, y fueron aun mayores cuando se usó una combinación de $100 \mathrm{mg}$ del extracto de $R$. graveolens / $\mathrm{kg} / \mathrm{día}$ y $100 \mathrm{mg}$ del dexametasona/kg/día.

En el grupo 2 se observó daño generalizado caracterizado por pérdida de la arquitectura normal del lobulillo hepático, congestión vascular y necrosis en los hepatocitos, así como signos sugestivos de apoptosis. Estos resultados se correlacionan con lo observado en un estudio previo de Roma et al. (2009), que describe que la dexametasona es capaz de inducir apoptosis. Sin embargo, otros estudios in vivo no reportan cambios morfológicos en hígado producidos por la administración de dexametasona (Kishida et al., 2008; Wang et al., 2011).

En este trabajo se observó que las ratas tratadas con 30 y $100 \mathrm{mg} / \mathrm{kg}$ de peso corporal con el extracto acuoso de $R$. graveolens ganaron el mismo peso que las del grupo control negativo. de Freitas et al. (2005), evaluaron el efecto producido por un extracto metanólico de $R$. graveolens administrado por 15 días vía oral en dosis de $1000 \mathrm{mg} / \mathrm{kg}$ en ratones, y al igual que en este estudio no se encontró variación significativa respecto al peso corporal. 
Por otra parte la relación peso corporal/peso de hígado se modificó con el tratamiento del extracto, este aumento en el peso puede ser atribuido a la esteatosis generalizada y/o a la presencia de inflamación causada por la liberación de componentes citoplásmicos de las células en necrosis en los grupos expuestos (Searle et al., 1982; Kerr et al., 1995).

Un hallazgo importante en el análisis histológico de los grupos 3, 4 y 5 fue la observación dosis-dependiente de signos de necrosis, tales como: extracción total o parcial del citoplasma y núcleos picnóticos en los hepatocitos. En estos grupos también se observaron signos sugestivos de apoptosis como condensación del citoplasma y de la cromatina sobre la envoltura nuclear (Kerr et al., 1972; Majno \& Joris, 1995; Erickson, 1997).

Es conveniente destacar que la observación de manera simultánea de necrosis, así como signos sugestivos de apoptosis, aunque son esencialmente dos tipos diferentes de muerte celular pueden tener traslapes en una respuesta celular ante un compuesto tóxico a la misma dosis administrada; y que la amplitud del daño que finalmente activará ambos tipos de muerte celular depende de factores toxicocinéticos, bioactivación y lesión específica (Raffray \& Cohen, 1997). Por lo que nuestros resultados sugieren que ambos tipos de muerte celular podrían ser inducidos por el extracto de $R$. graveolens, o bien por compuestos derivados de su metabolismo y que tengan como blanco al hígado por su actividad detoxificante (Al-Ali et al., 2005).

Las plantas medicinales son potencialmente hepatotóxícas como Larrea tridentata (Stickel et al., 2000) tal y como se reporta en este estudio. El efecto hepatotóxico observado en este trabajo, es distinto a lo descrito en un estudio previo en nuestro laboratorio; en donde no se observó daño morfológico en hígado por la exposición a $160 \mathrm{mg}$ de un extracto acuoso de plantas de la especie Lippia graveolens (SotoDomínguez et al., 2012). Cabe destacar que las especies $L$. graveolens y $R$. graveolens empleadas en ambos estudios se colectaron en la misma región del Norte de México, aunque en distintas épocas del año. Por lo que los diferentes resultados observados en estos estudios pueden además ser debido a que las plantas de esta especie muestran variaciones en la composición y cantidad de los metabolitos secundarios dependiendo de los factores climáticos, época de cosecha y el estado de crecimiento (Cimanga et al., 2002; Lecona-Uribe et al., 2003; Salgueiro et al., 2003; Hernández et al., 2009).

Por otra parte, las alteraciones histológicas en hígado se acentuaron en el grupo 4. En este grupo se observaron focos de infiltrados de células mononucleares, que pueden ser resultado de la liberación de factores quimioatrayentes que se liberan en las células necróticas (Searle et al.; Kerr et al., 1995).
Destaca además que en este grupo se observó un daño severo en los hepatocitos. Estas alteraciones pueden ser debido a múltiples constituyentes que produce la especie vegetal, entre los que se encuentran cumarinas y sus compuestos derivados (Orlita et al.; Ratheesh et al.). Se ha descrito que las cumarinas tienen como órganos blanco para su toxicidad y carcinogenicidad a hígado y pulmón en ratón y rata (Lake, 1999). También se han relacionado con afectación a la síntesis de los factores de coagulación en el hígado, además de ser causantes de hemorragias (Bye \& King, 1970, Pineda-Ruiz, 2009). Por lo que la presencia de cumarinas y otros compuestos aislados de $R$. graveolens podrían estar induciendo un efecto tóxico en los hepatocitos, congestión vascular, e infiltrados de células mononucleares observados en los grupos expuestos a distintas concentraciones del extracto de $R$. graveolens.

Las alteraciones observadas en los grupos 2, 3 y 4, fueron aun mayores en el grupo 5, posiblemente como resultado de un efecto aditivo o sinergista entre ambos (Han et al., 2011).

Los efectos tóxicos del extracto acuoso de $R$. graveolens sobre el hígado descrito previamente en el análisis histológico, se corroboraron en el análisis morfométrico, en donde se observó diferencia estadística significativa en los grupos 2, 3, 4 y 5, comparados con el grupo 1.

Actualmente en nuestro laboratorio se llevan a cabo estudios para identificar los compuestos químicos presentes en el extracto acuoso evaluado en este trabajo, así como la evaluación in vitro e in vivo de extractos metanólicos y aceites esenciales obtenidos de $R$. graveolens del norte de México.

\section{CONCLUSIONES}

Los hallazgos descritos en la presente investigación, demuestran que la exposición al extracto acuoso de $R$. graveolens, usada ampliamente en la medicina tradicional en México y diversos países, producen alteraciones histológicas severas en el hígado de ratas Wistar. Los productos a base de plantas son potencialmente dañinos, sobre todo cuando se desconoce la composición de los constituyentes tóxicos.

\section{AGRADECIMIENTOS}

Agradecemos el valioso apoyo en el manejo de animales a la MVZ Katty González Pineda y al CONACyT. 
SERRANO-GALLARDO, L. B.; SOTO-DOMÍNGUEZ, A.; RUIZ-FLORES, P.; NAVA-HERNÁNDEZ, M. P.; MORÁN-MARTÍNEZ, J.; GARCÍA-GARZA, R. \& MARTÍNEZ-PÉREZ, E. F. Efecto tóxico del extracto acuoso de Ruta graveolens del Norte de México sobre el hígado de rata wistar. Int. J. Morphol., 31(3): 1041-1048, 2013.

SERRANO-GALLARDO, L. B.; SOTO-DOMÍNGUEZ, A.; RUIZ-FLORES, P.; NAVA-HERNÁNDEZ, M. P.; MORÁNMARTÍNEZ, J.; GARCÍA-GARZA, R. \& MARTÍNEZ-PÉREZ, E. F. Toxic effect of aqueous extract of Ruta graveolens from the North of Mexico on the liver of Wistar rat. Int. J. Morphol., 31(3):1041-1048, 2013.

SUMMARY: In traditional medicine, beneficial properties are attributed to Ruta graveolens, but it has been shown that it can also cause side effects. Because there is no evidence that ratifies that $R$. graveolens is totally harmless. The aim of this study was to determine the toxic effect of aqueous extract of the leaves of this plant in rat liver. 25 Wistar rats were used and divided into 5 groups (n $=5$ ). Group 1 corresponded to the negative control group; group 2 or positive control was treated with $100 \mathrm{mg}$ of dexamethasone/kg/day. Groups 3 and 4 were treated with 30 and $100 \mathrm{mg}$ of extract of $R$. graveolens respectively. Group 5 was treated with $100 \mathrm{mg}$ dexamethasone/ $\mathrm{kg} /$ day in combination with $100 \mathrm{mg}$ of extract of $R$. graveolens $/ \mathrm{kg} / \mathrm{day}$. Administrations were performed intraperitoneally for three days. All animals were sacrificed by cervical dislocation. Liver samples were fixed in formalin and then embedded in paraffin. Histological sections were obtained from 5 microns thick and stained with hematoxylin-eosin for histological evaluation by light microscopy. The results demonstrate for the first time that exposure to aqueous extract of $R$. graveolens induces morphological changes in the liver of Wistar rats. These alterations were observed slightly in group 3. Alterations increased in group 4. Highest damage was observed in group 5 . The aqueous extract of $R$. graveolens was toxic; furthermore additional studies are needed to characterize other toxicological effects that support the risk of use of $R$. graveolens in traditional medicine in a long term.

KEY WORDS: Ruta graveolens; Aqueous extract; Liver toxicity; Wistar rats

\section{REFERENCIAS BIBLIOGRÁFICAS}

Agbonon, A.; Eklu-Gadegbeku, K.; Aklikokou, K.; Gbeassor, M.; Akpagana, K.; Tam, T. W.; Arnason, J. T. \& Foster, B. C. In vitro inhibitory effect of West African medicinal and food plants on human cytochrome P450 3A subfamily. J. Ethnopharmacol., 128(2):390-4, 2010.

Al-Ali, S. Y.; Hassan, I. M. \& Sadek, S. Ultrastructural changes in rat livers perfused in vitro and in vivo with a high dose of methotrexate. Histol. Histopathol., 20(4):1131-45, 2005.

Alonso-Castro, A. J.; Maldonado-Miranda, J. J.; Zarate-Martínez, A.; Jacobo-Salcedo, M. R.; Fernández-Galicia, C.; FigueroaZúñiga, L. A.; Ríos-Reyes, N. A.; de León-Rubio, M. A.; Medellín-Castillo, N. A.; Reyes-Munguía, A.; MéndezMartínez, R. \& Carranza-Álvarez, C. Medicinal plants used in the Huasteca Potosina, México. J. Ethnopharmacol., 143(1):292-8, 2012.

Bye, A. \& King, H. K. The biosynthesis of 4-Hydroxycoumarin and dicoumarol by Aspergillus fumigatus Fresenius. Biochem. J., $117(2): 237-45,1970$.

Cimanga, K.; Kambu, K.; Tona, L.; Apers, S.; De Bruyne, T.; Hermans, N.; Totté, J.; Pieters, L. \& Vlietinck, A. J. Correlation between chemical composition and antibacterial activity of essential oils of some aromatic medicinal plants growing in the Democratic Republic of Congo. J. Ethnopharmacol., 79(2):213-20, 2002.

de Freitas, T. G.; Augusto, P. M. \& Montanari, T. Effect of Ruta graveolens L. on pregnant mice. Contraception, 71(1):74-7, 2005.

Diwan, R. \& Malpathak, N. Furanocoumarins: novel topoisomerase I inhibitors from Ruta graveolens L. Bioorg. Med. Chem., 17(19):7052-5, 2009.
Diwan, R. \& Malpathak, N. Histochemical localization in Ruta graveolens cell cultures: elucidating the relationship between cellular differentiation and furanocoumarin production. Vitro. Cell Dev. Biol. Plant., 46(1):108-16, 2010.

Erickson, G. F. Defining apoptosis: players and systems. J. Soc. Gynecol. Invest. 4(5):219-28, 1997.

Freire, R. B.; Borba, H. R. \& Coelho, C. D. Ruta graveolens L. toxicity in Vampirolepis nana infected mice. Indian $J$. Pharmacol., 42(6):345-50, 2010.

González-Trujano, M. E.; Carrera, D.; Ventura-Martínez, R.; Cedillo-Portugal, E. \& Navarrete, A. Neuropharmacological profile of an ethanol extract of Ruta chalepensis L. in mice. J. Ethnopharmacol., 106(1):129-35, 2006.

Han, Y. L.; Yu, H. L.; Li, D.; Meng, X. L.; Zhou, Z. Y.; Yu, Q.; Zhang, X. Y.; Wang, F. J. \& Guo, C. Inhibitory effects of limonin on six human cytochrome P450 enzymes and Pglycoprotein in vitro. Toxicol. In Vitro, 25(8):1828-33, 2011.

Harat, Z. N.; Sadeghi, M. R.; Sadeghipour, H. R.; Kamalinejad, M. \& Eshraghian, M. R. Immobilization effect of Ruta graveolens L. on human sperm: a new hope for male contraception. J. Ethnopharmacol., 115(1):36-41, 2008.

Hernández, T.; Canales, M.; Ávila, J. G.; Garcia, A. M.; Meraz, S.; Caballero, J. \& Lira, R. Composition and antibacterial activity of essential oil of Lippia graveolens H.B.K. (Verbenaceae). Bol. Latinoam. Caribe Plant. Med. Aromat., 8(4):295-300, 2009.

Inamul, H. Safety of medicinal plants. Pakistan J. Med. Res., 43(4), 2004. 
SERRANO-GALLARDO, L. B.; SOTO-DOMÍNGUEZ, A.; RUIZ-FLORES, P.; NAVA-HERNÁNDEZ, M. P.; MORÁN-MARTÍNEZ, J.; GARCÍA-GARZA, R. \& MARTÍNEZ-PÉREZ, E F. Efecto tóxico del extracto acuoso de Ruta graveolens del Norte de México sobre el hígado de rata wistar. Int. J. Morphol., 31(3):1041-1048, 2013.

Kerr, J. F.; Gobé, G. C.; Winterfold, C. M. \& Harmon, B. V. Anathomical method in cell death. Methods Cell Biol., 46:127,1995 .

Kerr, J. F.; Wyllie, A. H. \& Currie, A.R. Apoptosis: a basic biological phenomenon with wide-ranging implications in tissue kinetics. Br. J. Cancer, 26(4):239-57, 1972.

Kishida, T.; Muto, S.; Hayashi, M.; Tsutsui, M.; Tanaka, S.; Murakami, M. \& Kuroda, J. Strain differences in hepatic cytochrome P450 1A and 3A expression between SpragueDawley and Wistar rats. J. Toxicol. Sci., 33(4):447-57, 2008.

Lake, B. G. Coumarin metabolism, toxicity and carcinogenicity: relevance for human risk assessment. Food Chem. Toxicol., 37(4):423-53, 1999.

Lecona-Uribe, S.; Loarca-Piña, F. G.; Arcila-Lozano, C.; DíazMoscoso, C. \& Ocampo, R. Nutraceutical potential of Mexican oregano (Lippia graveolens K). IFT Annual Meeting, 14E-28, 2003.

Libman, A.; Bouamanivong, S.; Southavong, B.; Sydara, K. \& Soejarto, D. D. Medicinal plants: an important asset to health care in a region of Central Laos. J. Ethnopharmacol., 106(3):303-11, 2006.

Majno, G. \& Joris, I. Apoptosis, oncosis, and necrosis. An overview of cell death. Am. J. Pathol., 146(1):3-15, 1995.

Oliva, A.; Meepagala, K. M.; Wedge, D. E.; Harries, D.; Hale, A. L.; Aliotta, G. \& Duke, S. O. Natural fungicides from Ruta graveolens L. leaves, including a new quinolone alkaloid. $J$. Agric. Food Chem., 51(4):890-6, 2003.

Orlita, A.; Sidwa-Gorycka, M.; Malinski, E.; Czerwicka, M.; Kumirska, J.; Golebiowski, M.; Loikowska, E. \& Stepnowski, P. Effective biotic elicitation of Ruta graveolens L. shoot cultures by lysates from Pectobacteriumatrosepticum and Bacillus sp. Biotechnol. Lett., 30(3):541-5, 2008.

Pineda-Ruiz, J. C. Sobreanticoagulación con warfarina. Arch. Med. (Manizales), 9(2):174-82, 2009.

Raffray, M. \& Cohen, G. M. Apoptosis and necrosis in toxicology: a continuum or distinct modes of cell death? Pharmacol. Ther., 75(3):153-77, 1997

Raghav, S. K.; Gupta, B.; Agrawal, C.; Goswami, K. \& Das, H. Anti-inflammatory effect of Ruta graveolens L. in murine macrophage cells. J. Ethnopharmacol., 104(1-2):234-9, 2006.

Rahim, F.; Saki, G. \& Bazrafkan, M. Effect of alcohol extracts of the Ruta graveolens $\mathrm{L}$. on the count, motility and in Vitro fertilization capacity of rats's sperm. Asian J. Plant. Sci., 9(1):63-6, 2010 .

Ratheesh, M.; Shyni, G. L. \& Helen, A. Methanolic extract of Ruta graveolens $\mathrm{L}$. inhibits inflammation and oxidative stress in adjuvant induced model of arthritis in rats. Inflammopharmacology, 17(2):100-5, 2009.

Roma, L. P.; Bosqueiro, J. R.; Cunha, D. A.; Carneiro, E. M.; Gurgul-Convey, E.; Lenzen, S.; Boschero, A. C. \& Souza, K. L. Protection of insulin-producing cells against toxicity of dexamethasone by catalase over expression. Free Radic. Biol. Med., 47(10):1386-93, 2009.

Salgueiro, L. R.; Cavaleiro, C.;Goncalves, M. J. \& Proenca da Cunha, A. Antimicrobial activity and chemical composition of the essential oil of Lippia graveolens from Guatemala. Planta Med., 69(1):80-3, 2003.

Seak, C. J. \& Lin, C. C. Ruta graveolens intoxication. Clin. Toxicol. (Phila.), 45(2):173-5, 2007.

Searle, J.; Kerr, J. F. \& Bishop, C. J. Necrosis and Apoptosis:Distinct modes of cell death with fundamentally different significance. Pathol. Annu., 17(Pt. 2):229-59, 1982.

Shanmugan, S.; Rajendran, K. \& Suresh, K. Traditional uses of medicinal plants among the rural people in Sivagangai district of Tamil Nadu, Southern India. Asian Pac. J. Trop. Biomed., 2(1):429-34, 2012

Soto-Domínguez, A.; García-Garza, R.; Ramírez-Casas, Y.; MoránMartínez, J. \& Serrano-Gallardo, L. B. El extracto acuoso de orégano (Lippia graveolens HBK) del norte de México tiene actividad antioxidante sin mostrar un efecto toxico in vitro e in vivo. Int. J. Morphol., 30(3):937-44, 2012.

Stickel, F.; Egerer, G. \& Seitz, H. K. Hepatotoxicity of botanicals. Public Health Nutr., 3(2):113-24, 2000.

Varamini, P.; Soltani, M. \& Ghaderi, A. Cell cycle analysis and cytotoxic potential of Ruta graveolens against human tumor cell lines. Neoplasma, 56(6):490-3, 2009.

Wang, X. \& Yeung, J. H. Inhibitory effect of tanshinones on rat CYP3A2 and CYP2C11 activity and its structure-activity relationship. Fitoterapia, 82(4):539-45, 2011.

Dirección para Correspondencia:

M.C. Edith Fabiola Martínez-Pérez

Departamento de Bioquímica y Farmacología

Centro de Investigación Biomédica

UA de C., Unidad Torreón

Av. Gregorio A. García 198 Sur

Torreón, Coah. C.P. 27000

MÉXICO

Email: edith_fabiolamartinez@hotmail.com

Recibido : 12-01-2013

Aceptado: 19-05-2013 